\title{
Plastic deformation of solid-solution strengthened Hf-Nb-Ta-Ti-Zr body-centered cubic medium/high-entropy alloys
}

\author{
Rajeshwar R. Eleti*, Nikita Stepanov, Nikita Yurchenko, Denis Klimenko, Sergey Zherebtsov \\ Laboratory of Bulk Nanostructured Materials, Belgorod National Research University, Belgorod 308015, Russia
}

\section{A R T I C L E I N F O}

\section{Article history:}

Received 20 March 2021

Revised 9 April 2021

Accepted 10 April 2021

Available online 24 April 2021

\section{Keywords:}

High-entropy alloys

Mechanical behavior

Stress equivalence

Activation volume

Screw dislocations

\begin{abstract}
A B S T R A C T
Plastic deformation and rate-controlling mechanism during tensile deformation of various body-centered cubic (BCC) medium/high-entropy alloys (MEAs/HEAs) were investigated at $300 \mathrm{~K}$. Microstructural observations revealed long, straight screw dislocations, occasionally consisting of jogs. The deformed substructures were consistent with the evaluated activation volume $\left(V^{*}\right)$ indicating screw dislocation motion overcoming the Peierls-Nabarro stress barrier as the rate-controlling mechanism. The $V^{*}$ values were found inversely proportional to the yield strength. The monotonous behavior of the $V^{*}$ as a function of yield strength indicated the dislocation motion of BCC-MEAs/HEAs must involve overcoming the energy barrier involving multiple/group of substitutional solute atoms, simultaneously. Furthermore, for the first time, stress equivalence was observed in the BCC-MEAs/HEAs. The findings described thermally-activated dislocation glide mechanisms of the solid-solution strengthened BCC-MEAs/HEAs depended on the stress rather than the chemical complexity of the alloys.
\end{abstract}

(C) 2021 Acta Materialia Inc. Published by Elsevier Ltd. All rights reserved.
The plastic deformation of metallic materials is primarily driven by dislocation motion. Dislocations, however, must overcome the maximum energy barrier to account for the plastic strain, $\varepsilon$. The transition rate of the dislocations at this barrier can be expressed as [1];

$\dot{\varepsilon}=\dot{\varepsilon}_{0} \exp (-Q / k T)$

where, $\dot{\varepsilon}$ is the strain rate, $\dot{\varepsilon}_{0}$ is a pre-exponential constant, $Q$ is the activation energy, $k$ is Boltzmann's constant, $T$ is the temperature. The rate-equation (Eq. 1) indicates that dynamics of plastic strain is a thermally-activated process. The activation energy for a thermally-activated dislocation glide is, however, not a constant value, but depends on the applied stress. The general feature is that the activation energy to overcome the barrier decreases with an increase of the applied stress, thus directly affects the activation volume $\left(V^{*}\right)$ under the operative limits of the same rate-controlling mechanism.

Screw dislocations glide is often found dominant in the bodycentered cubic (BCC) crystals [2,3]. Due to the non-planar core structure of the $a / 2<111>$ screw dislocations, it has been suggested that the screw dislocations overcoming the Peierls-Nabarro stress barrier controls the plastic flow rate [4]. At ambient or sub-zero temperatures, however, BCC alloys often show the transformation

\footnotetext{
* Corresponding author at: Laboratory of Bulk Nanostructured Materials, Belgorod National Research University, Pobeda 85, Belgorod, 308015, Russia.

E-mail address: rajeshwar.eleti@gmail.com (R.R. Eleti).
}

of Peierls-type to kink/kink-pair nucleation mechanism which impacts the fundamental mechanism of dislocation motion. Such a transformation is regarded as the transformation of a non-planar dislocation core structure into a planar, as was initially found by Vitek [5].

In the new class of crystalline materials like medium/highentropy alloys (MEAs/HEAs) [6-8], the lack of compositional periodicity in the lattice may lead to randomly distributed local Peierls-Nabarro stress field having different rates of energy changes. Rao et al. [9] studied various BCC-MEAs using atomistic simulations and first-principles calculations and found corestructure variation along the screw dislocation. Their investigations revealed the NbTiZr BCC-MEA showed extended screw dislocation core structure along multiple $\{110\}$ slip-planes, similar to BCC metals and alloys. It is rather intriguing to verify experimentally the validity of the fundamental rate-controlling mechanism in the BCCMEAs/HEAs, given the richness of the solid-solution strengthening (SSS) of these alloys. Considering the vast compositional space of MEAs/HEAs, it is possible to fabricate a series of appropriate alloys having various strengths or flow behaviors, which would allow the investigation of thermally activated rate-controlling mechanisms at different yield/flow stresses. By doing so, not only the rate-controlling mechanism will be determined, but better understanding of the nature of dislocation-solute(s) interactions in the BCC-MEAs/HEAs can be obtained. Therefore, in the present study, we chose different BCC-MEAs/HEAs having various compositions and investigated the effect of stress on the rate-controlling mecha- 
nisms by qualitative analysis. Similar experiments were performed on pure $\mathrm{Nb}$ as a case reference. Besides, the rate-controlling mechanisms were discussed using relevant microstructural investigations.

The vacuum arc-melted $\mathrm{Nb}$, equiatomic NbTiZr, HfNbTa, HfTaTiZr, and HfNbTaTiZr alloys were cold-rolled until $65 \sim 86 \%$ height reduction and annealed at $0.5 \mathrm{~T}_{\mathrm{m}}\left(\mathrm{T}_{\mathrm{m}}\right.$ is the melting temperature) for $20 \mathrm{~min}$ and subsequently water quenched (for more details on thermo-mechanical processing, refer to the Supplementary materials, S1). Phase identification of the recrystallized specimens was performed using the X-ray diffraction (XRD) technique. Microstructures were investigated using transmission electron microscope (TEM) and field emission-scanning electron microscope (FE-SEM) equipped with a back-scattering electron (BSE) imaging system. Tensile specimens having gauge dimensions $4 \times 1 \times 0.8 \mathrm{~mm}^{3}$ were cut from the annealed sheets and tested at room temperature (RT) at a constant strain-rate $10^{-3} \mathrm{~s}^{-1}$. Here, it is noteworthy that we evaluated the rate-controlling mechanisms by determining the activation volume $\left(V^{*}\right)$ using the stress relaxation test during tensile deformation. The $V^{*}$ was calculated by using the following equation [4],

$\Delta \sigma=\frac{-M k_{B} T}{V^{*}\left[1+\frac{\theta}{E}\right]} \ln \left(1+\frac{t}{C}\right)$

where, $\Delta \sigma$ is change in the flow stress, $M$ is Taylor factor (2.7), $k_{\mathrm{B}}$ is Boltzmann's constant, $T$ is temperature, $V^{*}$ is apparent activation volume, $\theta$ is strain hardening rate, $E$ is elastic modulus of machine-specimen assembly, $t$ is time, $C$ is a time constant, where $C=M k T /\left(V^{*} E \dot{\varepsilon}_{p}^{0}\right)$ which can be deduced from the natural logarithmic plot of Eq. 2.

Fig. 1(a) shows structural characterization of different alloys using XRD. XRD profiles indicated that all the alloys had a singlephase BCC structure. Fig. 1(b-f) shows SEM-BSE images of Nb and different BCC-MEAs/HEAs. The BSE images revealed that all alloys were fully recrystallized and had a uniform grain size in the range of $18-80 \mu \mathrm{m}$. Fig. 2(a) shows engineering tensile stress-strain curves of the program alloys. As is clear, the yield strength increased with an increase of the number of elements from pure $\mathrm{Nb}$ to the equiatomic HfNbTaTiZr HEA. It should be noted, however, the yield strengths of the 3 and 4-component HfNbTa and HfTaTiZr MEAs, respectively, were very close to that of the 5-component HfNbTaTiZr HEA.

It is noteworthy that Coury et al. [10] reported an elevated thermal stress component contribution to the yield strength of the BCC-HEAs at RT. Here, in order to predict the yield strengths of the present BCC-MEAs/HEAs at RT, we have performed theoretical estimations of the yield strength in a high-throughput manner similar to the report of Coury et al. [10]. The details of determining the yield strength were shown in the Supplementary materials, S2 and the results are shown in Fig. 2(b). Based on the theoretical estimations, it was clear that the experimentally observed yield strengths of the present BCC-MEAs/HEAs were higher than the predicted yield strengths of the alloys. However, the trend of strength increasing was correctly predicted by the performed calculations.

Further, stress relaxation tests were performed during tensile deformation to evaluate the activation volume $\left(V^{*}\right)$. Fig. 3(a) shows the stress $v s$. time curves of various alloys during the stress relaxation tests. The details on the evaluation of $V^{*}$ were shown in the Supplementary materials, S3. Fig. 3(b) shows the evolution of $V^{*}$ as a function of strain.

The evaluated $V^{*}$ of $\mathrm{Nb}$ precisely at the yield strength showed $V^{*} \sim 109 \mathrm{~b}^{3}$; this value then slightly increased before saturation at $\sim 140 \mathrm{~b}^{3}$. The obtained $V^{*}$ values of the NbTiZr and HfNbTa alloys ranged $47 \sim 41 \mathrm{~b}^{3}$ and $35 \sim 30 \mathrm{~b}^{3}$, respectively. Finally, the $V^{*}$ values of the HfTaTiZr alloy ranged $33 \sim 31 \mathrm{~b}^{3}$. In our previous article [11], a similar range of the $V^{*}$ values $\left(31 \sim 29 \mathrm{~b}^{3}\right)$ was observed in the HfNbTaTiZr alloy. The $V^{*}$ values of the alloys in the present study were mostly strain independent, which is due to low strain hardening rate of these alloys. However, the $V^{*}$ values of the NbTiZr and HfNbTa alloys decreased slightly with strain reflecting the minor strain hardening of the stress-strain curves shown in Fig. 2(a).

The evaluated $V^{*}$ indicated thermally-activated dislocation glide by overcoming the Peierls-Nabarro stress barrier as the ratecontrolling mechanism. It is noteworthy that the $V^{*}$ values of all the alloys were well above the range of kink/kink-pair nucleation, $V^{*}<10 \mathrm{~b}^{3}$ [11]. That is, independent of the alloy composition or tensile strength, the $V^{*}$ values indicated no relevant transition from the Peierls-type to kink nucleation mechanism. Furthermore, the $V^{*}$ values became equal when the yield/flow stresses of different alloys became equal (i.e., independent of the chemical composition of the alloys or number of elements present in the alloy); compare the data for HfNbTa and HfTaTiZr in Fig. 3, respectively, for example. This is referred to as the stress equivalence [12]. Fig. 4 shows the effect of yield strength on $V^{*}$.

As is clear, the $V^{*}$ value decreased with an increase of the yield stress by $(\sigma)^{-1.05}$. The linear relationship between $V^{*}$ and stress of Fig. 4, suggested that only one rate-controlling mechanism operated in the entire stress range of the present MEAs/HEAs. The important and noticeable considerations from Fig. 3 and Fig. 4 can be characterized as:

\section{$1 V^{*}$ decreases with an increase of yield strength.}

2 When the yield/flow stress of two alloys of different chemical composition becomes equal, the $V^{*}$ values of those alloys also becomes equal (i.e., stress equivalence).

Firstly, as the stress increased, $V^{*}$ decreased. This is because, at high stresses according to the Peierls-Nabarro model, the high energy (metastable) state and the equilibrium state of the dislocation line in the force-distance curve become very close to each other. Due to this, the work done by the applied stress against the thermally-activated dislocation barrier increases; consequently, the length of the dislocation segment activated facing the barrier decreases. Thus, the $V^{*}$ value decreases as a function of stress. This means, although a large number of elements in the MEAs/HEAs may enhance the strength due to SSS, the present results indicated the overall scheme of the Peierls-Nabarro model does not change for the MEAs/HEAs in terms of dislocation glide mechanism.

Friedel [13] made independent statistical reports on the strong dislocation-solute interaction model for the SSS mechanisms. Accordingly, the model predicts $V^{*} \propto(\sigma)^{-n}$, where the stress exponent $n=2$. On the other hand, Basinski et al. [12] performed a systematic study on the face-centered cubic (FCC) Cu-based alloys and found a lower stress exponent, $n \leq 1$, as in a weak dislocationsolute interaction model of Labusch $[14,15]$. Recently, Kawamura et al. [16] found the $V^{*}$ values of the CoCrFeMnNi FCC-HEA linearly scaled with the $\mathrm{Cu}$ and Ag-based FCC dilute alloys. Our present observations on the concentrated BCC alloys indicated the stress exponent of $n \sim 1.05$, which was surprisingly close to that of the FCC alloys. According to Kocks [17], Mott and Nabarro [18], and Labusch $[14,15]$ the resulted tendency of $V^{*}$ as a function of stress points that the solid-solution of present BCC-MEAs/HEAs composed of many week and few, if not nil strong obstacles against the initiation of dislocation motion. This would mean, the overall thermallyactivated dislocation segment must overcome several soluted elements for a successful activation step. Such a viewpoint would facilitate the possibility of stress equivalence, as was initially found by Basinski et al. [12], and later reviewed by Nabarro [13] and Kocks, Argon, and Ashby [19]. Based on these observations, it can be suggested that the thermally-activated dislocation glide resistance in the present solid-solution strengthened BCC-MEAs/HEAs is most likely controlled by the energy barrier overcoming mul- 

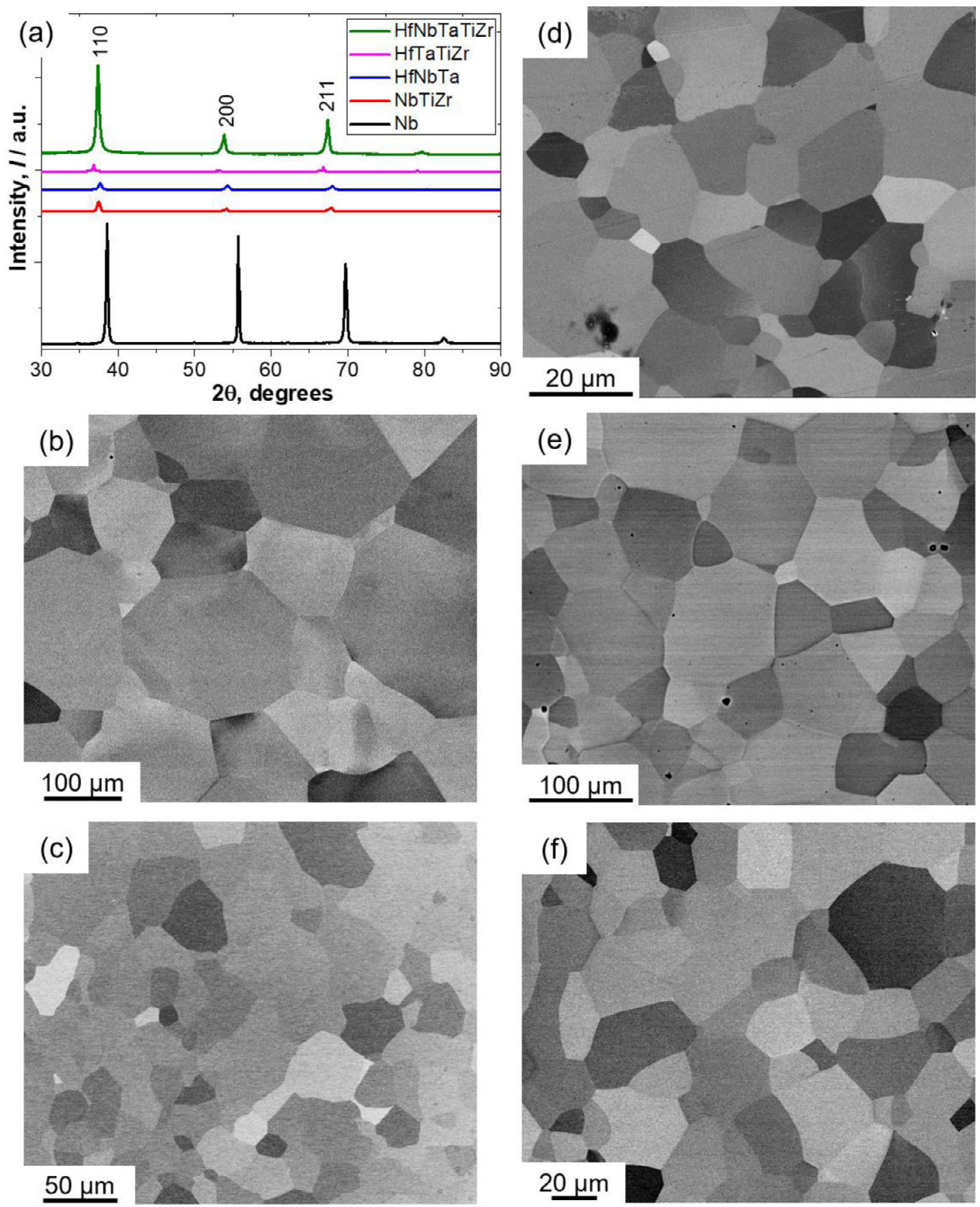

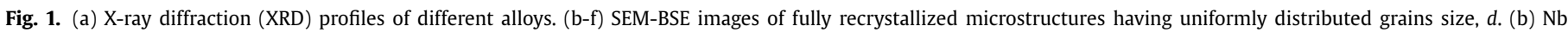
( $d \sim 80 \mu \mathrm{m}),(\mathrm{c}) \operatorname{NbTiZr}(d \sim 55 \mu \mathrm{m})$, (d) HfNbTa $(d \sim 18 \mu \mathrm{m})$, (e) HfTaTiZr $(d \sim 40 \mu \mathrm{m})$, (f) HfNbTaTiZr $(d \sim 60 \mu \mathrm{m})$.

tiple substitutional solute atoms together. The implications of the present results highlight the SSS mechanism of the present BCCMEAs/HEAs is largely due to the dislocation-solute(s) elastic interaction, which can be attributed to the atomic size and modulus misfits of the constituent elements of the HEAs. The current phenomenological viewpoint can qualitatively validate the present experimental findings like the decrease of $V^{*}$ by increasing the yield strength and the possibility of stress equivalence between different alloys.

Although several theories has been suggested for the origin of high yield strengths of the HEAs [20-25], one of the compelling reasonings was provided by Okamoto et al. [26]. They found that the yield strengths of the FCC-HEAs linearly corre- lated with the mean-square atomic displacement (MSAD) of the HEAs, without using additional scaling parameters. Later, Nohring and Curtin [27] also showed correlations for the yield strengths as a function of micro/atomic-distortions in the FCC and BCC concentrated alloys. In our present study, the theoretically estimated yield strength of the alloys were determined using the atomic size and modulus differences between the individual elements that constitute for the lattice distortion. Although the predicted yield strength of the alloys were lower than the experimentally observed data, the trend in experimental values was correctly predicted. All of these above reports describes, though less certain, that the dislocation-solute(s) interaction of the HEAs is largely elastic and supports the possibility that a 
(a)

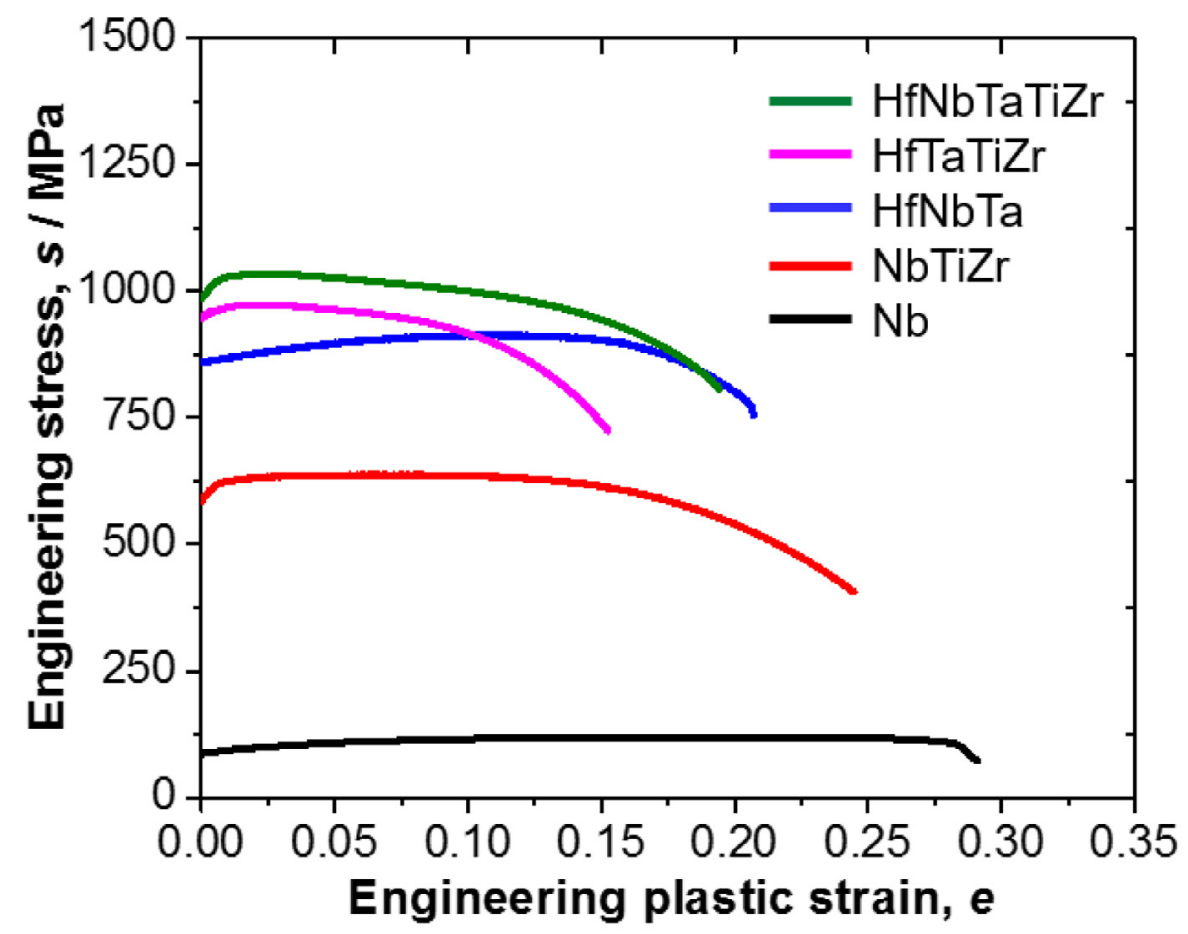

(b)

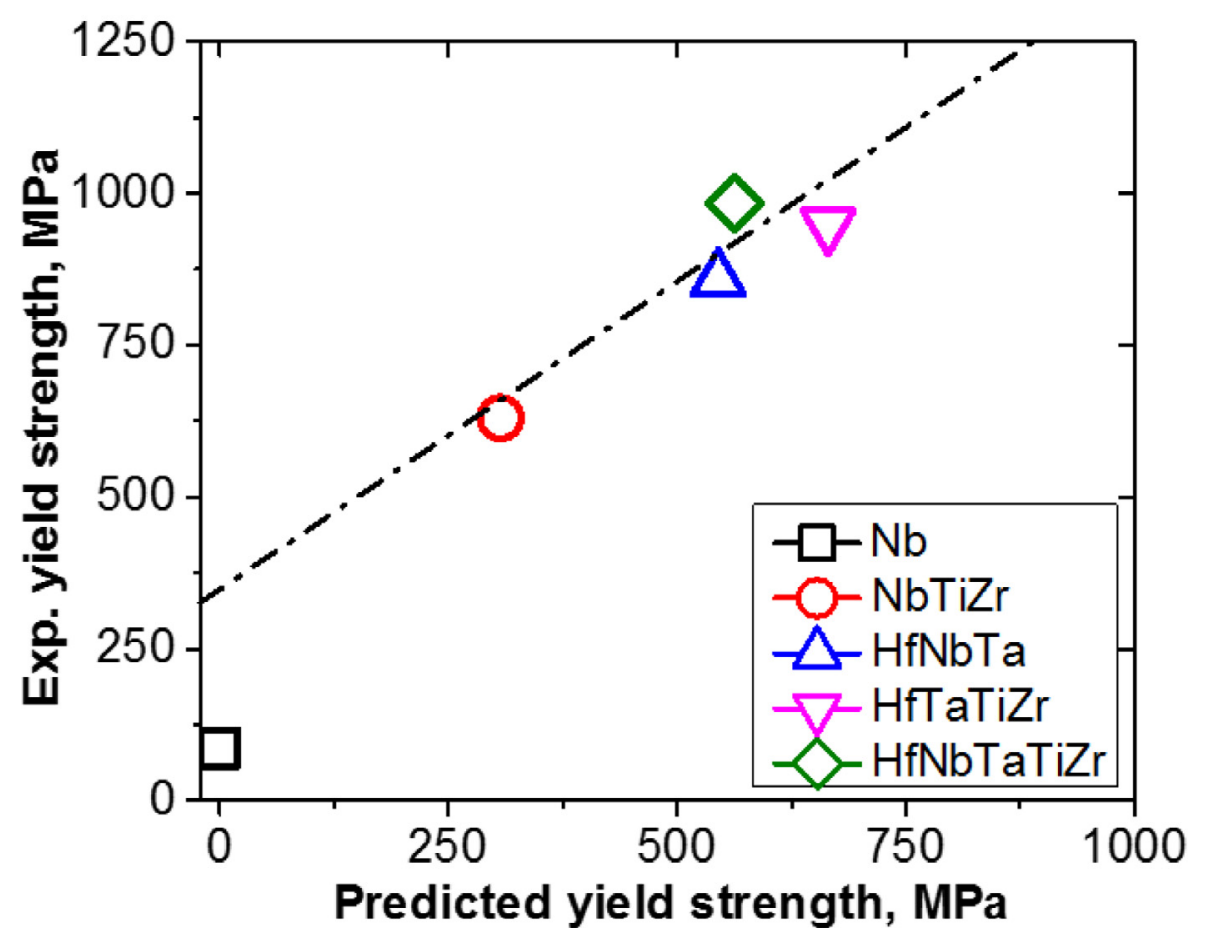

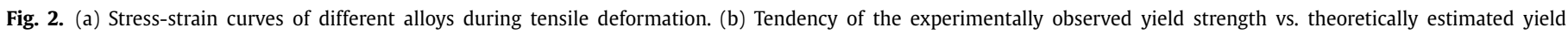
strength of various BCC-MEAs/HEAs.

unit thermally-activated dislocation motion would consist of multiple substitutional solute(s) atoms. That is, the thermally activated rate-controlling mechanisms of the BCC-MEAs/HEAs depends on the applied stress rather than the chemical complexity of the alloys.
To access the plastic flow mechanisms and verify the relevance of $V^{*}$ values, investigations of the deformation substructures were performed using TEM. Here, it is noteworthy that the stress-strain curve of the HfNbTa MEA (Fig. 2(a)) showed indications of slight strain hardening which merits further investigation. However, here 
(a)

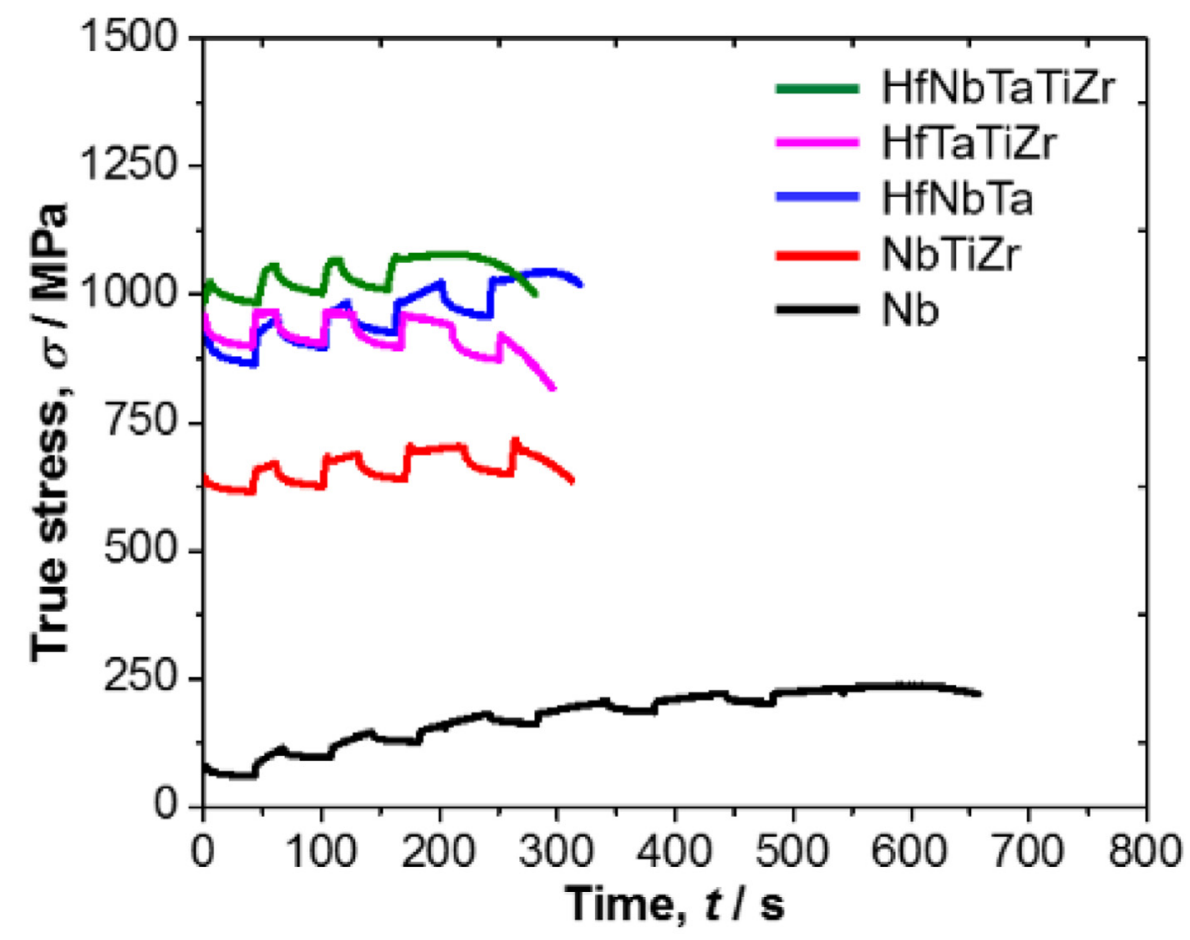

(b)

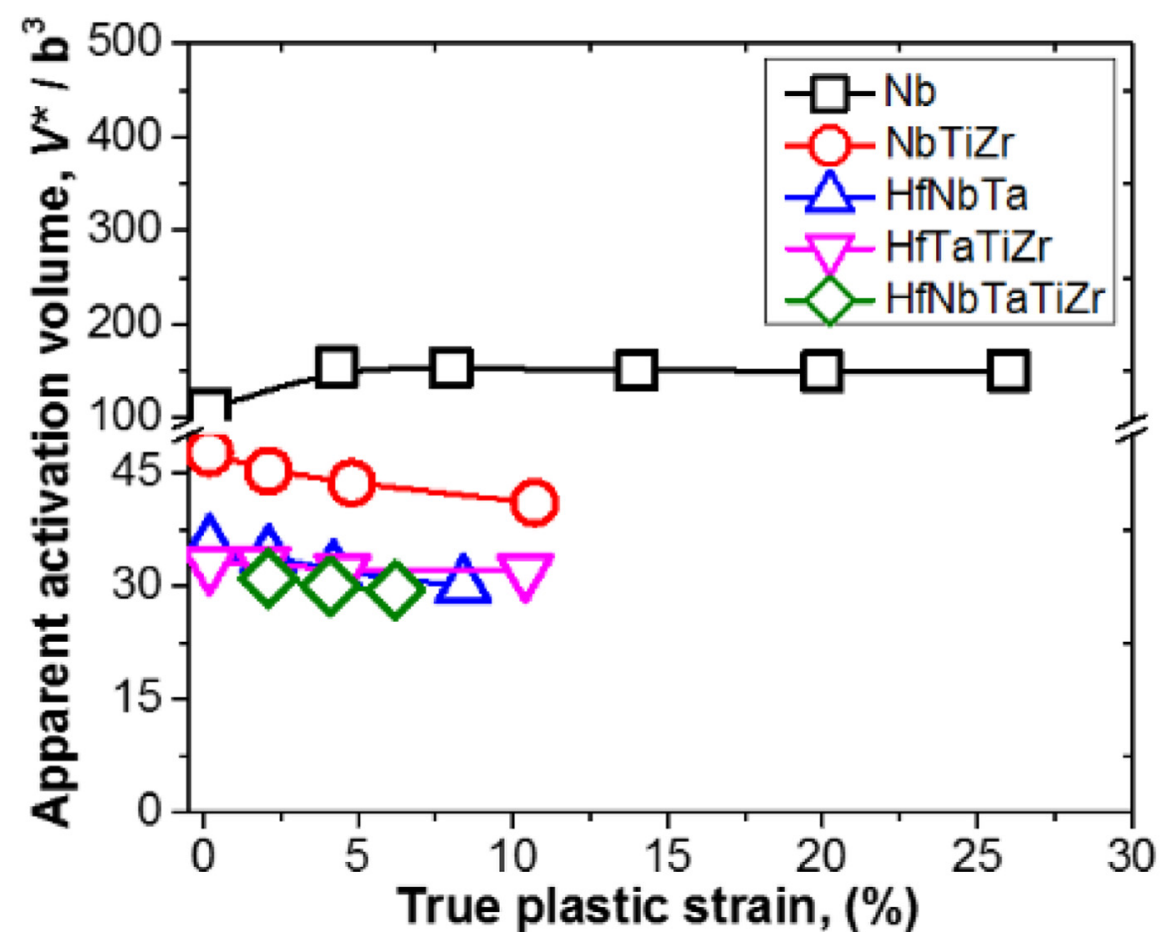

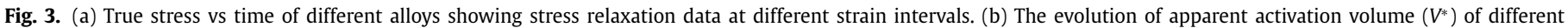
alloys as a function of deformation strain.

we chose the NbTiZr MEA and HfNbTaTiZr HEA, as both of them were lying on either end of the stress-range spectrum among the program alloys. As shown in Fig. 5, TEM images of NbTiZr and HfNbTaTiZr indicated long, straight screw dislocations were dominantly observed in both the alloys at the early stages of plastic flow (Fig. 5(a,b)) [also refer to the Supplementary materials, S3], as well as after fracture (Fig. 5(c-f)). The observed long, and straight dislocation morphology similar to BCC metals and alloys indicated that the dislocations of BCC-MEAs/HEAs lacked the dislocation configuration preferences introduced by the large number of elements 


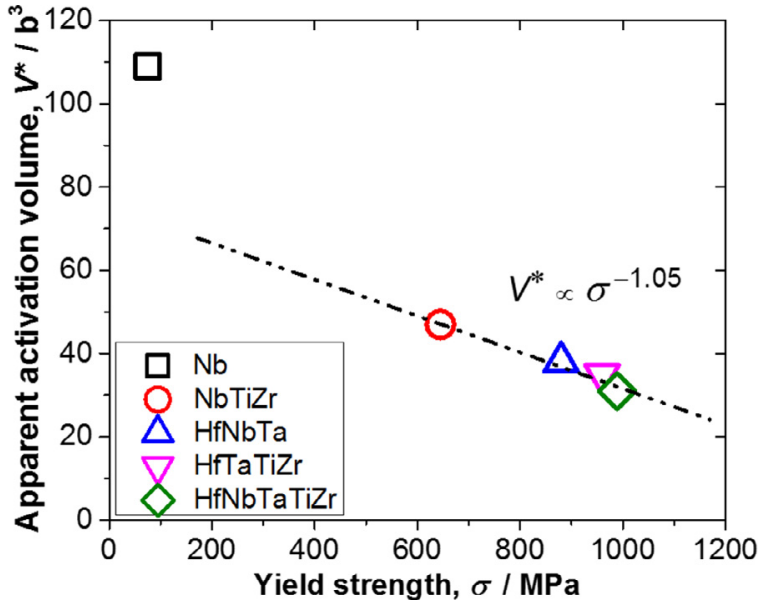

Fig. 4. Variation in the activation volume $\left(V^{*}\right)$ as a function of yield strength of different alloys at $300 \mathrm{~K}$. [ $\sigma$ is yield strength measured at $\sigma_{0.2 \%}$.].

[28]. In other words, this also suggested that the chemical bond part is less relevant as long as it does not change the flexibility of the dislocations. Furthermore, dislocation jogs were frequently observed on the long, straight screw dislocations. Jogs, in general, can be formed either due to dislocations intersection or during cross-slip of a screw dislocation from the primary slip plane [4]. Moreover, jogs on the screw-dislocations have the edge dislocation configuration. Due to this, screw dislocation glide may generate a trail of dipoles, which may facilitate the formation of dislocation loops, as was observed in Fig. 5. Identical dislocations dipole debris during screw dislocation glide was also found in the atomistic simulation studies of BCC-MEAs/HEAs [9,25]. Dislocations structure like jogs that can provide additional glide resistance unfortunately does not enter into the theoretical estimation of the yield strength. The observed higher experimental yield strengths than the predicted strengths (Fig. 2(b)) can be due to the formation of jogs. However, the density of jogs in both the alloys were too low to have a critical effect on the rate-controlling mechanisms during plastic flow. Moreover, the observation of long, straight screw dislocations in the early stages of the plastic flow has displayed thermally-activated dislocation motion overcoming Peierls-Nabaro stress barrier (friction stress) in both the alloys, similar to a BCC metal, Vanadium found by Christian et al. [28]. Therefore, in the context of the above observations, screw dislocation motion overcoming the Peirls-Nabarro stress barrier controls the rate of plastic flow in the present alloys.

Lastly, some of the recent investigations have suggested the significance of edge dislocations mobility in the BCC-HEAs [29-32]. Li et al. [33] reported that the strength of edge dislocations of NbMoTaW BCC-HEA showed $20 \%$ of the strength of screw dislocations. According to their findings, however, the ratio of screw/edge dislocations mobility was still too large for the edge dislocations to have a critical effect on the fundamentals of plastic deformation of the BCC-HEAs. Couzinie et al. [34] studied the microstructure evolution of the HfNbTaTiZr BCC-HEA and showed that the alloy deformed predominantly by the screw dislocations glide, as was observed in the present study. Moreover, the data provided in Fig. 2(b) using the fitting parameter $\alpha=12.2$ (refer, the Supplementary materials, S2) has indicated a better representation of the present experimental data suggesting dominant screw dislocations glide. Therefore, the present results indicated that not all BCC-HEAs are susceptible to a high edge dislocations mobility, at least at RT.

To summarize, the mechanical properties of different BCCMEAs/HEAs was systematically investigated during tensile deformation. The yield strength of the alloys tended to increase with the number of components, yet this dependence wasn't linear. The dislocation motion of different alloys was controlled by a single thermally-activated dislocation glide mechanism, i.e. overcoming the Peierls-Nabarro stress barrier. Further, stress equivalence, i.e. identical values of activation volume in different alloys at the same

\section{$\mathrm{NbTiZr}$}

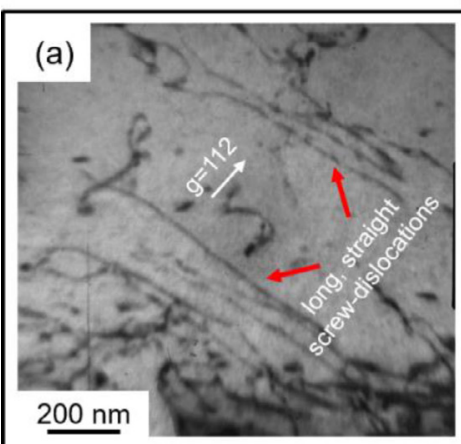

(b)

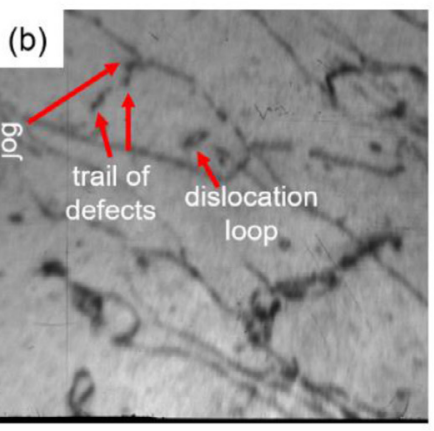

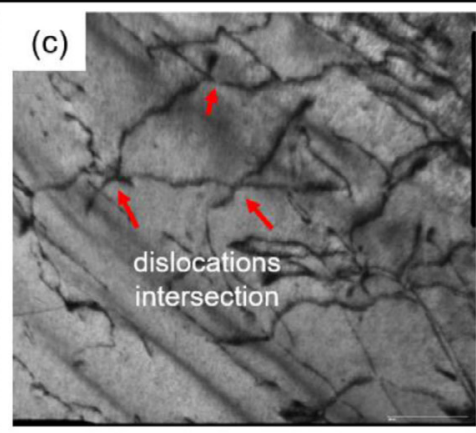

\section{(d)}

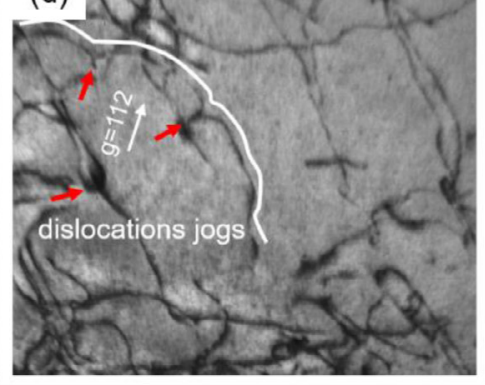

$\mathrm{HfNbTaTiZr}$

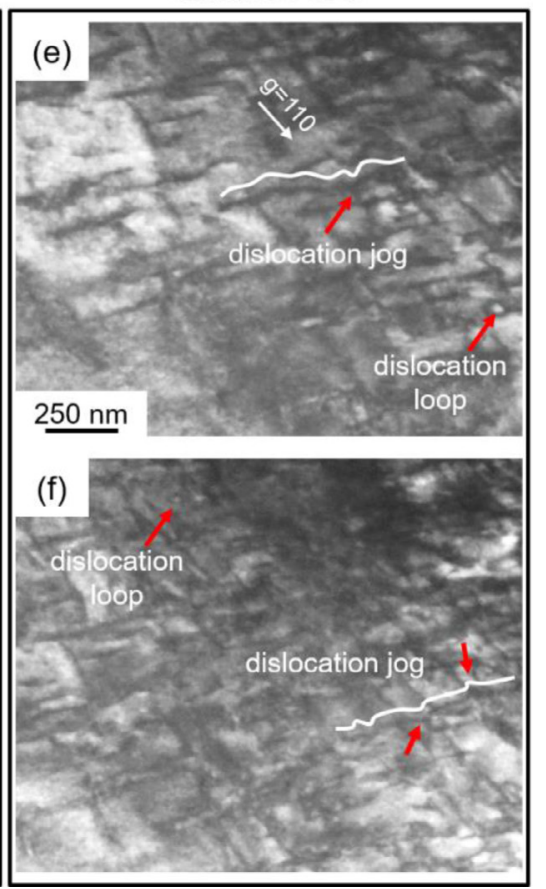

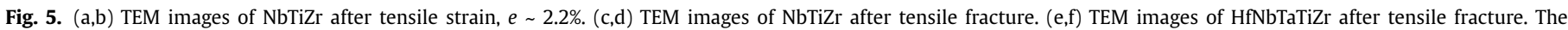

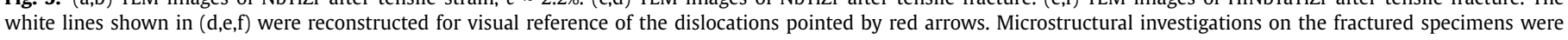
performed away from the localized (necking on tensile specimen) deformation region. 
yield yield stresses was found between alloys with various chemical compositions and a number of components. Microstructural investigations of the deformed substructure revealed screw dislocations, suggesting that the plastic flow dominantly occurred by the screw dislocations glide. It was rather conclusive that the fundamental dislocation motion in the present BCC-MEAs/HEAs was governed by the thermally-activated rate-controlling mechanisms particularly dependent on the applied stress.

\section{Declaration of Competing Interest}

The authors declare that they have no known competing financial interests or personal relationships that could have appeared to influence the work reported in this paper.

\section{Acknowledgements}

The authors gratefully acknowledge the financial support from the Russian Science Foundation Grant no. 19-79-30066. The authors are grateful to the personnel of the Joint Research Center, "Technology and Materials", Belgorod National Research University, for their assistance. The authors would like to extend acknowledgements to Dr. D. Shaysultanov of Laboratory of Bulk Nanostructured Materials, Belgorod National Research University for assistance with the melting of the alloys.

\section{Supplementary materials}

Supplementary material associated with this article can be found, in the online version, at doi:10.1016/j.scriptamat.2021. 113927.

\section{References}

[1] H. Conrad, JOM 16 (1964) 582-588.

[2] C.R. Weinberger, B.L. Boyce, C.C. Battaile, Int. Mater. Rev. 58 (2013) 296-314.
[3] D. Caillard, Acta Mater 161 (2018) 21-34.

[4] D. Caillard, J.L. Martin, Thermally Activated Mechanisms in Crystal Plasticity, first ed., Pergamon, 2003.

[5] V. Vítek, Phys. Status Solidi 18 (1966) 687-701.

[6] J.-W. Yeh, S.-K. Chen, S.-J. Lin, J.-Y. Gan, T.-S. Chin, T.-T. Shun, C.-H. Tsau, S.-Y. Chang, Adv. Eng. Mater. 6 (2004) 299-303.

[7] M.-H. Tsai, J.-W. Yeh, Mater. Res. Lett. 2 (2014) 107-123.

[8] D.B. Miracle, O.N. Senkov, Acta Mater 122 (2017) 448-511.

[9] S.I. Rao, B. Akdim, E. Antillon, C. Woodward, T.A. Parthasarathy, O.N. Senkov, Acta Mater 168 (2019) 222-236.

[10] F.G. Coury, M. Kaufman, A.J. Clarke, Acta Mater 175 (2019) 66-81.

[11] R.R. Eleti, N. Stepanov, S. Zherebtsov, Scr. Mater. 188 (2020) 118-123.

[12] Z.S. Basinski, R.A. Foxall, R. Pascual, Scr. Metall. 6 (1972) 807-814

[13] F.R.N. Nabarro, Proc. R. Soc. London. A. Math. Phys. Sci. 381 (1982) 285-292.

[14] R. Labusch, Phys. Status Solidi 41 (1970) 659-669.

[15] R. Labusch, Acta Metall 20 (1972) 917-927.

[16] M. Kawamura, M. Asakura, N.L. Okamoto, K. Kishida, H. Inui, E.P. George, Acta Mater 203 (2021) 116454.

[17] U.F. Kocks, Metall. Trans. A 16 (1985) 2109-2129.

[18] F.R.N. Nabarro, Phys. Soc. London 75 (1948).

[19] U.F. Kocks, A.S. Argon, M.F. Ashby, Thermodynamics and Kinetics of Slip, Pergamon, 1975.

[20] H. Chen, A. Kauffmann, S. Laube, I.-C. Choi, R. Schwaiger, Y. Huang, K. Lichtenberg, F. Müller, B. Gorr, H.-J. Christ, M. Heilmaier, Metall. Mater. Trans. A 49 (2018) 772-781.

[21] Q.-J. Li, H. Sheng, E. Ma, Nat. Commun. 10 (2019) 3563.

[22] C. Varvenne, A. Luque, W.A. Curtin, Acta Mater 118 (2016) 164-176.

[23] L. Zhang, Y. Xiang, J. Han, D.J. Srolovitz, Acta Mater 166 (2019) 424-434.

[24] S.I. Rao, E. Antillon, C. Woodward, B. Akdim, T.A. Parthasarathy, O.N. Senkov, Scr. Mater. 165 (2019) 103-106.

[25] F. Maresca, W.A. Curtin, Acta Mater 182 (2020) 144-162.

[26] N.L. Okamoto, K. Yuge, K. Tanaka, H. Inui, E.P. George, AIP Adv 6 (2016) 125008

[27] W.G. Nöhring, W.A. Curtin, Scr. Mater. 168 (2019) 119-123.

[28] A. Christian, O. Kanert, J.T.. De Hosson, Acta Metall. Mater. 38 (1990) 2479-2484.

[29] F. Maresca, W.A. Curtin, Acta Mater 182 (2020) 235-249.

[30] F. Maresca, C. Lee, R. Feng, Y. Chou, T. Ungar, M. Widom, K. An, J. Poplawsky, Y.-C. Chou, P. Liaw, W. Curtin, Edge Dislocations Can Control Yield Strength in Refractory Body-Centered-Cubic High Entropy Alloys, 2020.

[31] L.T.W. Smith, Y. Su, S. Xu, A. Hunter, I.J. Beyerlein, Int. J. Plast. 134 (2020) 102850.

[32] F. Wang, G.H. Balbus, S. Xu, Y. Su, J. Shin, P.F. Rottmann, K.E. Knipling, J.-C. Stinville, L.H. Mills, O.N. Senkov, I.J. Beyerlein, T.M. Pollock, D.S. Gianola, Science (80-. ) 370 (2020) 95 LP -101.

[33] X.-G. Li, C. Chen, H. Zheng, Y. Zuo, S.P. Ong, Npj Comput. Mater. 6 (2020) 70

[34] J.-P. Couzinié, L. Lilensten, Y. Champion, G. Dirras, L. Perrière, I. Guillot, Mater. Sci. Eng. A 645 (2015) 255-263. 